\title{
Models of Epidemics and Endemicity in Genetically Variable Host Populations*
}

Ira M. Longini, Jr.

Departments of Statistics and Epidemiology, University of Michigan, Ann Arbor, MI 48109, USA

\begin{abstract}
A discrete time genetics model is developed for populations that are undergoing selection due to infectious disease. It is assumed that the generation time of the host and infectious agent are non-synchronous and that only the host population is evolving. Two classes of epidemic processes are considered. The first class is for infectious agents that confer immunity following infection, while the second class is for those that do not confer immunity. The necessary and sufficient conditions are found in order for the disease to persist in a stable polymorphic host population. These conditions are shown to depend on the density of susceptibles, the selection coefficients, and the severity and class of the disease process.
\end{abstract}

Key words: Endemicity - Epidemics - Genetics - Deterministic models Stability

\section{Introduction}

A proposition of considerable epidemiological importance is that host genetic variability is partially responsible for the maintenance of infectious disease in populations. Moreover, the proposition that infectious diseases act as important selective forces for maintaining polymorphisms in host populations is an intriguing part of theories of evolution. Epidemiologists have observed that human populations vary considerably in both susceptibility and response to infection, and have suggested that there may be genetic mechanisms acting. The most striking example is the resistance of erythrocytes containing $\mathrm{HbS}$ (i.e., the sickle-cell trait) to infection by Plasmodium falciparum malaria parasites [7] and the resistance of the Duffy-blood-group-negative erythrocytes, FyFy, to infection by Plasmodium vivax malaria parasites $[14,15]$. With regard to host response to infection, there is generally indirect evidence of genetic variation. Such an effect may be demonstrated by the marked variation among children in the severity of diseases produced by highly infectious organisms such as chicken pox. Specific resistance genes for infectious agents affecting humans have been located in mice. These agents include

\footnotetext{
* This research was partially supported by the National Institute of Health under National Research
} Service Award AI-6103-01 
the etiological agents of visceral leishmaniasis and scrub typhus [17]. Mice have also demonstrated marked genetic variation in susceptibility to salmonella infections. Bremermann [4] has hypothesized that the high degree of polymorphisms of host defenses may be a strategy of dealing with myriad evolving pathogens.

The co-evolution of host and pathogen may be important in maintaining polymorphisms in both groups. Several authors have developed mathematical models of the co-evolution of pathogens and their hosts [10,20]. Lewis [12] gives an excellent summary of this work as well as new results. Most of these models have been limited to the case where the pathogen and host have the same generation time. These "synchronized" generation models are inappropriate for most human and animal diseases because while the generation time of the host is on the order of many years, that of the pathogen may be only a matter of a few days. Therefore, a model which allows for non-synchronous generations is needed.

Gillespie [8] developed a model for non-synchronous populations involving those infectious diseases which confer immunity following infection and where only the host evolves. Lewis [12] analyzed Gillespie's model for haploid populations and gave the sufficient conditions for maintaining a stable polymorphism in the host. Kemper [11] has worked with epidemic models for diseases that do not confer immunity following infection and where both genetic and epidemic components are continuous in time. The purpose of this paper is to extend the work of Gillespie and Lewis to account for a wider variety of infectious disease processes, including those which do not confer immunity following infection. Except for malaria, no attempt is made to relate the results of this paper directly to the diseases described above. However, the approach and a generalization of the results presented here should prove useful in understanding other specific disease-gene interactions.

\section{Basic Genetics Model}

An important assumption is that the generation time of the host is much longer than that of the pathogen. The host has discrete, non-overlapping generations such that the epidemic process runs its course within each host generation. Analysis will be limited to single-locus diallelic populations. Since the qualitative results are the same for the haploid and diploid populations considered in this paper, the simpler haploid population will be presented first. It is assumed that there is no migration or mutation and that the population size is large (conceptually infinite). Let the two alleles $\mathbf{A}$ and $\mathbf{a}$ occur with frequencies $p$ and $q=1-p$. Then, using standard population genetic arguments (e.g., Crow and Kimura, $1970:$ p. 179), the frequency of $\mathbf{A}$ in the next generation will be

$$
p^{\prime}=\frac{p w_{1}}{\bar{w}},
$$

where $\bar{w}=p w_{1}+q w_{2}$. The $w_{i}$ 's represent relative fitnesses that depend on the level of infection during the current generation. The level of infection $\mathscr{I}[\bar{k}(p), \sigma]$ is defined as the probability that a genetically susceptible individual is infected (or chronically infected, depending on the epidemic process envisaged) during a generation. The function $\bar{k}(p)$ is defined as the average density of the susceptible 
population,

$$
\bar{k}(p)=k_{1} p+k_{2} q
$$

where $0 \leqslant k_{1} \leqslant 1$ and $0 \leqslant k_{2} \leqslant 1$ are the coefficients of relative susceptibility of the two genotypes. The constant $\sigma$ is the infectious contact parameter, which will be specified in Sect. 3. If it is assumed that the a genotype is totally resistant to infection (i.e., $k_{2}=0$ ), then

$$
\begin{aligned}
w_{1} & =(1-\mathscr{I}) v_{1}+\mathscr{I} r_{1} \\
& =v_{1}-\mathscr{I}\left(v_{1}-r_{1}\right), \quad 0 \leqslant r_{1}<v_{1} \leqslant 1,
\end{aligned}
$$

and

$$
w_{2}=v_{2}, \quad 0 \leqslant v_{2} \leqslant 1
$$

The $v_{i}$ 's are the relative fitnesses of non-infected individuals, while $r_{1}$ is the relative fitnesses of infected individuals of genotype $\mathbf{A}$. Without loss of generality, we allow a susceptible (but not infected) individual of genotype $\mathbf{A}$ to have unit fitness; thus,

$$
v_{1}=1, \quad r_{1}=1-t \quad \text { and } \quad v_{2}=1-s,
$$

where $s$ and $t$ are selection coefficients. The relative fitnesses are now

$$
w_{1}=1-\mathscr{I} t \quad \text { and } \quad w_{2}=1-s .
$$

The relative fitness $w_{1}$ is an implicit function of $p$ and, therefore, selection is frequency dependent. Cockerham et al. (1972) give a general description of frequency dependent selection.

Equation (1) is easily solved for the equilibrium gene frequency $p^{*}$. We are interested in the polymorphic equilibrium (i.e., $0<p^{*}<1$ ), which implies

$$
w_{1}=\bar{w}=w_{2}
$$

Solving Eq. (1) at $0<p^{*}<1$ yields

$$
\mathscr{I}\left[\bar{k}\left(p^{*}\right), \sigma\right]=\frac{s}{t} .
$$

We see that $\mathscr{I}^{*}$ (the infection rate for susceptibles) depends only on the selection coefficients and is independent of the infection process. However, the value of $p^{*}$ does depend on the epidemic process used. A necessary condition for a polymorphic equilibrium to exist is that $0<s<r \leqslant 1$, since we require that $0<\mathscr{I}<1$. Therefore, an infected individual of genotype $\mathbf{A}$ is less fit than a resistant individual of genotype a who, in turn, is less fit than a susceptible individual of genotype $\mathbf{A}$.

A polymorphic equilibrium is locally asymptotically stable (LAS) if

$$
-1<\left.\frac{\partial p^{\prime}}{\partial p}\right|_{*}<1
$$

where

$$
\left.\frac{\partial p^{\prime}}{\partial p}\right|_{*}=1+\left.\frac{p}{\bar{w}}\left[\frac{\partial w_{1}}{\partial p}-\frac{\partial \bar{w}}{\partial p}\right]\right|_{*}
$$


Equations (4), (5), (6) and (7) are combined to yield the conditions for $p^{*}$ to be LAS, that is

$$
-1<1-\left.\left[\frac{p q t}{1-s}\right] \mathscr{I}^{\prime}\right|_{*}<1
$$

where $\mathscr{I}^{\prime}=d \mathscr{I} / d p$. The right-hand side of inequality (8) is satisfied if

$$
\left.\frac{p q t}{1-s} \mathscr{I}^{\prime}\right|_{*}>0
$$

This is true if $\left.\mathscr{I}^{\prime}\right|_{*}>0$. Therefore, $\mathscr{I}$ must be a monotone increasing function of $p$ in the neighborhood of $p^{*}$. The left-hand side of inequality (8) is satisfied if

$$
\left.\frac{p q t}{1-s} \mathscr{I}^{\prime}\right|_{*}<2
$$

In general, inequality (10) will be satisfied as long as the slope of $\mathscr{I}$ is not too great in the neighborhood of $p^{*}$.

\subsection{Diploid Populations}

Now consider a one locus, diallelic diploid host population. There are three genotypes: AA, Aa and aa. Equation (1) remains the same, where

$$
\begin{aligned}
& w_{1}=p w_{11}+q w_{12}, \\
& w_{2}=p w_{12}+q w_{22}, \quad 0 \leqslant w_{i j} \leqslant 1,
\end{aligned}
$$

and $w_{11}, w_{12}$ and $w_{22}$ are the relative fitnesses of the three genotypes $\mathbf{A A}, \mathbf{A a}$ and $\mathbf{a a}$, respectively. Equation (2) gives the average density of the susceptible population, where

$$
\begin{aligned}
& k_{1}=p k_{11}+q k_{12}, \\
& k_{2}=p k_{12}+q k_{22}, \quad 0 \leqslant k_{i j} \leqslant 1 .
\end{aligned}
$$

Two cases are considered here. The first is when the susceptible allele $\mathbf{A}$ is completely recessive (i.e., $k_{12}=k_{22}=0$ ) and the second is when the susceptible allele $\mathbf{A}$ is completely dominant (i.e., $k_{22}=0$ ).

For the completely recessive case,

$$
\begin{aligned}
& w_{11}=1-\mathscr{I} t, \\
& w_{12}=w_{22}=1-s .
\end{aligned}
$$

At $0<p^{*}<1$, we again have

$$
\mathscr{I}\left[\bar{k}\left(p^{*}\right), \sigma\right]=\frac{s}{t} .
$$

Equations (4), (5), (6) and (12) are combined to yield the conditions for $p^{*}$ to be LAS, which are that

$$
-1<1-\left.\left[\frac{p^{2} q t}{1-s}\right] \mathscr{I}^{\prime}\right|_{*}<1 .
$$


Inequality (13) differs from (8) by a factor of $p$ and will be satisfied as long as the slope of $\mathscr{I}$ is not too great in the neighborhood of $p^{*}$.

In the case of complete dominance, the relative fitnesses are

$$
\begin{aligned}
& w_{12}=w_{11}=1-\mathscr{I} t \\
& w_{22}=1-s .
\end{aligned}
$$

Equations (4), (5), (6) and (14) are combined to yield the conditions for $p^{*}$ to be LAS, which are that

$$
-1<1-\left.\left[\frac{p q^{2} t}{1-s}\right] \mathscr{I}^{\prime}\right|_{*}<1,
$$

which differs from (8) by a factor of $q$ and will be satisfied under the same conditions stated above.

The results of this section can be summarized in the following theorem:

Theorem 1. For the single locus, diallelic populations specified above, the necessary conditions for the equilibrium point $0<\mathscr{I}^{*}<1,0<p^{*}<1$ to exist and be LAS are:

(a) $0<s<t \leqslant 1$

(b) $\mathscr{I}^{\prime}>0$ in the neighborhood of $p^{*}$,

(c) The slope of $\mathscr{I}$ is not too great in the neighborhood of $p^{*}$.

Theorem 1 gives the necessary conditions for a stable polymorphism to exist, while the sufficient conditions will be found by specifying the form of $\mathscr{I}$. Conditions (b) and (c) constrain $\mathscr{I}$ to those forms which make biological sense. Condition (b) requires that the level of infection in the population increases with the density of susceptibles. However, condition (c) dictates that this level of infection cannot be too sensitive to small changes in the density of susceptibles.

\section{Models of Infection}

In this section, two basic models that have become paradigms of infectious disease are described. The first is the so-called $S-I-S$ epidemic process, where infected individuals return to the susceptible state following their infection, i.e., there is no lasting immunity following infection. Diseases following this pattern generally become endemic. Examples of diseases that roughly follow this pattern are gonorrhea, meningitis, rhinovirus (common cold) and, to some extent, malaria. The second is the $S-I-R$ epidemic process, where infected individuals become immune or resistant to further infection. Examples of diseases that roughly follow this pattern are measles, mumps, chicken pox, polio and a single strain of influenza.

\subsection{The S-I-S Epidemic Process}

The population is partitioned into three mutually exclusive classes of individuals: susceptible (but not infected), infected and genetically resistant. Let $S(\tau)$ and $I(\tau)$ be the fractions of the genetically susceptible population in the susceptible and infected classes at time $\tau$, respectively. Since generations are discrete and non-overlapping, the population is closed for each generation. Mixing between susceptibles and 
infectives is homogeneous and the infectious contact rate $\beta$ is defined as the number of contacts per infective per unit of time. Infected individuals recover and are removed from the infected class at a rate $\gamma$ proportional to the number of infectives.

From the above description, the differential equations for the $S-I-S$ process are

$$
\begin{gathered}
S^{\prime}(\tau)=-S \bar{k}(p) \beta I+\gamma I, \\
I^{\prime}(\tau)=S \bar{k}(p) \beta I-\gamma I, \\
S(0)=S_{0}>0, \quad I(0)=I_{0}>0, \quad S(\tau)+I(\tau)=1,
\end{gathered}
$$

where $\beta>0$ and $\gamma>0$. Since it is assumed that the generation time of the host is much longer than that of the pathogen, it is expected that the epidemic process will approach its asymptotic values well within one host generation. The asymptotic behavior of $I(\tau)$ for large $\tau$ is as follows

$$
\lim _{\tau \rightarrow \infty} I(\tau)= \begin{cases}0 & \text { if } \quad \bar{k}(p) \sigma \leqslant 1, \\ 1-\frac{1}{\bar{k} \sigma} & \text { if } \quad \bar{k}(p) \sigma>1,\end{cases}
$$

where $\sigma=\beta / \gamma$ is the infectious contact number [9].

If $k \sigma>1$ at the beginning of a generation, the fraction of the population that is infected at any time $\tau$, when $\tau$ is large, will be $1-(1 / k \sigma)$. If an individual were randomly selected from the genetically susceptible population at time $\tau$, the probability that the individual would be infected is approximated by $1-(1 / \bar{k} \sigma)$. Therefore, the probability $\mathscr{I}$ is

$$
\mathscr{I}[\vec{k}(p), \sigma]=\lim _{\tau \rightarrow \infty} I(\tau)
$$

and

$$
\mathscr{I}^{\prime}=\frac{\bar{k}^{\prime}}{\bar{k}^{2} \sigma}, \quad \text { where } \quad \bar{k}^{\prime}=\frac{d \bar{k}(p)}{d p} .
$$

In haploid and diploid populations, we specify that $k_{2}=0$ and $k_{22}=0$, respectively. Then, it follows that

$$
\mathscr{I}^{\prime}>0 \quad \text { for } \quad 0<p<1 \text {, }
$$

and condition (b) of Theorem 1 is satisfied for the $S-I-S$ epidemic process.

\subsection{The S-I-R Epidemic Process}

The population is now partitioned into four mutually exclusive classes of individuals: susceptible, infected, immune following infection and genetically resistant. Let $S(\tau), I(\tau)$ and $R(\tau)$ be the fractions of the genetically susceptible population in the first three classes, respectively.

The differential equations for the $S-I-R$ process are

$$
\begin{aligned}
S^{\prime}(\tau) & =-S \bar{k}(p) \beta I, \\
I^{\prime}(\tau) & =S \bar{k}(p) \beta I-\gamma I, \\
R^{\prime}(\tau) & =\gamma I
\end{aligned}
$$




$$
\begin{gathered}
S(0)=S_{0}>0, \quad I(0)=I_{0}>0, \quad R(0)=0, \\
S(\tau)+I(\tau)+R(\tau)=1 .
\end{gathered}
$$

These differential equations have also been analyzed by others [2, 9]. The asymptotic behavior of $S(\tau), I(\tau)$ and $R(\tau)$ for large $\tau$ is summarized as

$$
\lim _{\tau \rightarrow \infty} I(\tau)=0, \quad \lim _{\tau \rightarrow \infty} S(\tau)=S_{\infty}>0 \quad \text { and } \quad \lim _{\tau \rightarrow \infty} R(\tau)=R_{\infty}>0,
$$

where $S_{\infty}$ is the unique root in $(0,1 / \bar{k} \sigma)$ of the equation

$$
S_{\infty}=S_{0} \exp \left[-\bar{k} \sigma\left(1-S_{\infty}\right)\right]=0 .
$$

If it is assumed that the epidemic process starts with a chance introduction of infection at the beginning of the generation, we allow $I_{0} \approx 0$ and $S_{0} \approx 1$. Then $R_{\infty}$ represents the fraction of individuals infected during the course of one generation. Substituting into Eq. (21) yields

$$
1-R_{\infty}-\exp \left[-\bar{k} \sigma R_{\infty}\right]=0 .
$$

No explicit solution is available, but $R_{\infty}$ can be found using successive approximation or some other iterative procedure.

The probability that a randomly selected individual will be infected during the course of one generation is approximated by $R_{\infty}$. Therefore, the probability $\mathscr{I}$ is found by solving the following implicit function

$$
\mathscr{I}[\bar{k}(p), \sigma]= \begin{cases}0 & \text { if } \quad \bar{k}(p) \sigma \leqslant 1, \\ 1-\exp (-\bar{k} \sigma \mathscr{I}) & \text { if } \quad \bar{k}(p) \sigma>1 .\end{cases}
$$

Then $\mathscr{I}^{\prime}$ is differentiated as an implicit function, yielding

$$
\begin{aligned}
\mathscr{I}^{\prime} & =\bar{k}^{\prime} \sigma \mathscr{I}^{2}(1-\mathscr{I})[\mathscr{I}+(1-\mathscr{I}) \ln (1-\mathscr{I})]^{-1} \\
& =\bar{k}^{\prime} \sigma \mathscr{I}^{2}(1-\mathscr{I})\left[\sum_{i=2}^{\infty} \frac{1}{i(i-1)} \mathscr{I}^{i}\right]^{-1},
\end{aligned}
$$

which lies in the open interval $(0,1-(1 / \bar{k} \sigma))$. ( $\mathscr{I}$ is not differentiable at $\bar{k} \sigma=1$. Evaluation of (24) for permissible values of $\bar{k}$ yields

$$
\mathscr{I}^{\prime}>0 \text { for } 0<p<1 \text {, }
$$

and condition (b) of Theorem 1 is satisfied for the $S-I-R$ epidemic process.

When interpreting the meaning of $\mathscr{I}$, for both the $S-I-S$ and $S-I-R$ processes, a word of caution is in order. Since $\mathscr{I}$ is defined as the probability that a genetically susceptible individual is infected during a generation, it does not refer to the whole population. If we define $\mathscr{F}$ as the probability that an individual sampled from the whole population (including genetically resistant individuals) is infected during a generation, then we have

$$
\mathscr{F}=\bar{k}(p) \mathscr{I} .
$$

Kemper [11] carefully makes this distinction and points out that would most likely be the "observable" measure of prevalence in field data. 


\section{Analysis of Genetic-Epidemic Model}

\subsection{Haploid Populations}

Assume that individuals of genotype $\mathbf{A}$ are completely susceptible (i.e., $k_{1}=1$ ), then from (2), the average density of susceptibles is

$$
\bar{k}(p)=p \text {. }
$$

For the $S$ - $I-S$ epidemic process, the probability $\mathscr{I}$ is given by (18) as

$$
\mathscr{I}(p, \sigma)= \begin{cases}0 & \text { if } p \sigma \leqslant 1 \\ 1-\frac{1}{p \sigma} & \text { if } p \sigma>1 .\end{cases}
$$

A plot of $\mathscr{I}$ as a function of $p$ is given in Fig. 1. The polymorphic equilibrium is found from (5) and (25) to be

$$
\mathscr{I}^{*}=s / t, \quad p^{*}=\frac{t}{(t-s) \sigma} .
$$

In general, we require that $\sigma>1$, since $p^{*}=1$ is LAS when $\sigma \leqslant 1$. Condition (a) in Theorem 1 guarantees that $p^{*}$ will be contained in the interval $(0,1)$ as long as $s$ is not too close to $t$.

Evaluation of (19) yields

$$
\mathscr{I}^{\prime}=\frac{1}{p^{2} \sigma}, \quad \text { when } \quad p \sigma>1 .
$$

Substituting Eq. (27) into Eq. (10) yields

$$
q^{*}\left(\frac{t-s}{1-s}\right)<2
$$

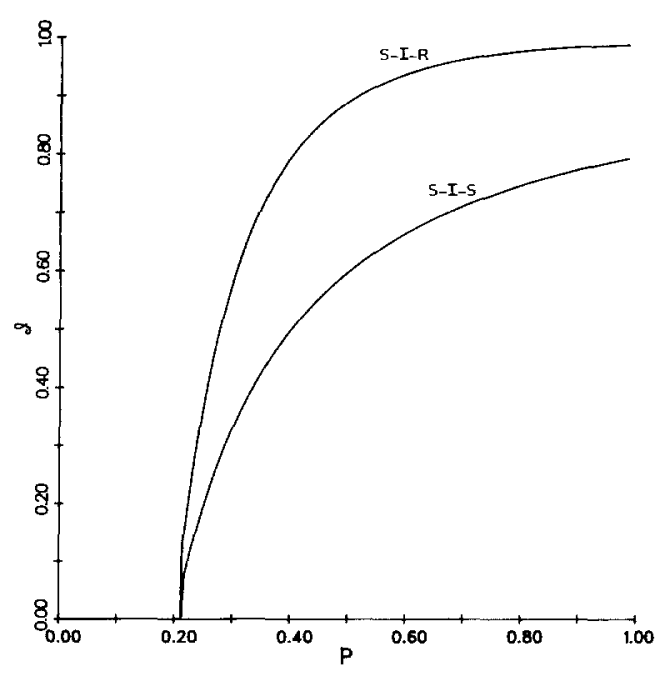

Fig. 1. Graph of $\mathscr{I}$ as a function of $p$ for a haploid population, where $\sigma=5$. Note that the more volatile $S-I-R$ epidemic process has a greater slope than the $S-I-S$ epidemic process 
which is satisfied by condition (a) of Theorem 1 . Therefore, the polymorphic equilibrium (26) exists and is LAS if $p^{*} \sigma>1$ and $0<s<t \leqslant 1$, where $s$ is not too close to $t$. Figure 2(a) shows different stable equilibrium points for various values of $s / t$ and $\sigma$.

Kemper [11] has shown that the polymorphic equilibrium (26) is LAS, in the case of continuous time, under very similar conditions to those given in Theorem 1. He has also ruled out the existence of limit cycles for the continuous case.
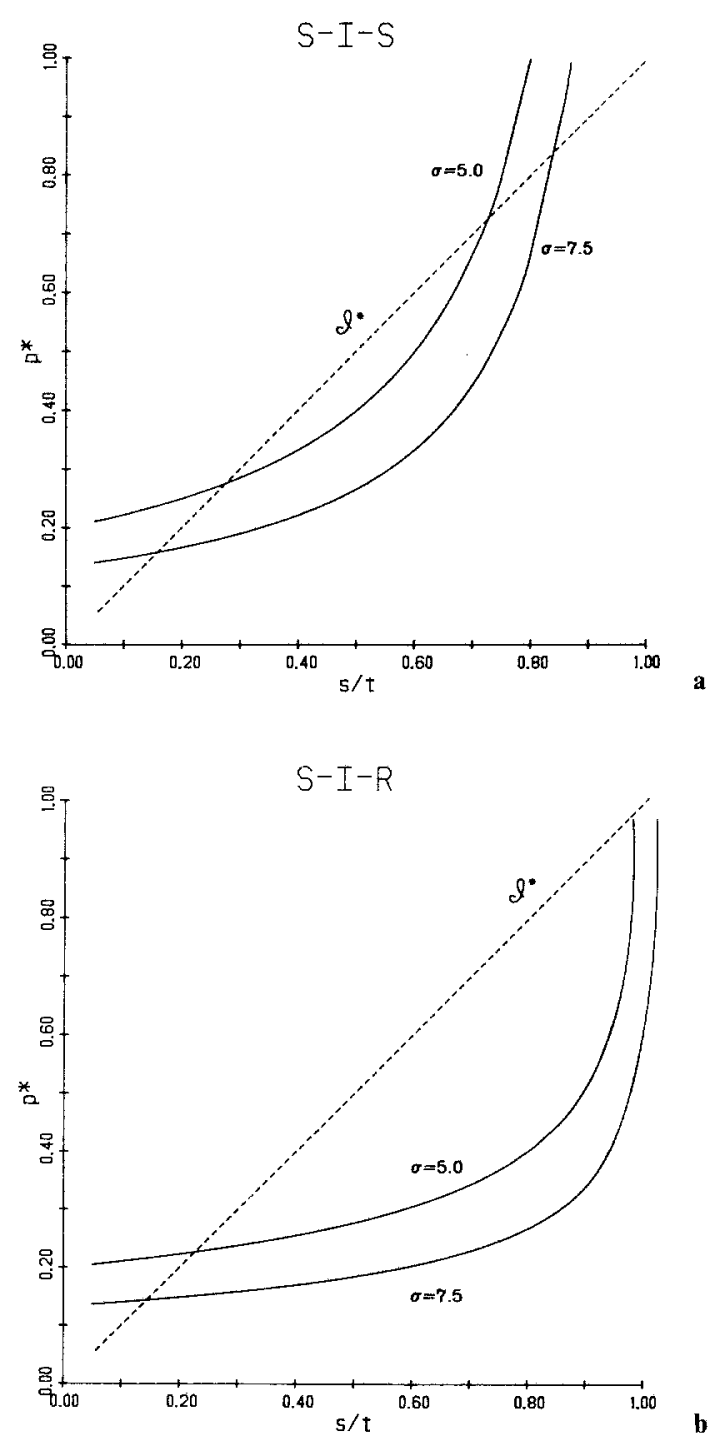

Fig. 2. Graph of relationship of the polymorphic equilibrium values $\mathscr{I}^{*}$ and $p^{*}$ at different ratios of selection coefficients for haploid populations. Note that the level of $p^{*}$ decreases with increasing values of $\sigma$. Also, $p^{*}$ is maintained at a lower level for the $S-I-R$ epidemic process than for the $S-I-S$ epidemic process, at the same parameter values 
In general, if $\sigma$ is not close to 1 , the disease will not die out. To see this, assume that at the beginning of generation $t$,

$$
p_{t} \sigma \leqslant 1 \text {. }
$$

Since $s<1$, it follows that

$$
p_{t+1}>p_{t}
$$

The value of $p$ will continue to increase with each generation until the equilibrium value is obtained, at which juncture $p^{*} \sigma>1$.

For the $S-I-R$ epidemic process, the probability $\mathscr{I}$ is given by (23) as

$$
\mathscr{I}(p, \bar{\sigma})= \begin{cases}0 & \text { if } p \sigma \leqslant 1 \\ 1-\exp (-p \sigma \mathscr{I}) & \text { if } p \sigma>1\end{cases}
$$

A plot of $\mathscr{I}$ as a function of $p$ is given in Fig. 1. The polymorphic equilibrium is found from (5) and (28) as

$$
\mathscr{I}^{*}=s / t, \quad p^{*}=\frac{-\ln \left(1-\mathscr{I}^{*}\right)}{\sigma \mathscr{I}^{*}}
$$

provided that $s$ is not too close to $t$. Evaluation of $(24)$ at $\bar{k}^{\prime}=1$, yields

$$
\mathscr{I}^{\prime}=\sigma \mathscr{I}^{2}(1-\mathscr{I})[\mathscr{I}+(1-\mathscr{I}) \ln (1-\mathscr{I})]^{-1} \text {. }
$$

Examination of Fig. 1 reveals that the slope of $\mathscr{I}$ is quite large, which implies that condition (c) of Theorem 1 may not be satisfied for all $0<s<t \leqslant 1$.

Substituting (30) into (10) yields

$$
\left.\frac{-q \mathscr{I}(1-\mathscr{I}) \ln (1-\mathscr{I})}{((1 / t)-\mathscr{I})[\mathscr{I}+(1-\mathscr{I}) \ln (1-\mathscr{I})]}\right|_{*}<2 .
$$

The left-hand side of (31) is a function of $s, t$ and $\sigma$, designated $f(s, t, \sigma)$, since $\mathscr{I} *$ is a function of $s$ and $t$ and $q^{*}$ is a function of $\mathscr{F}^{*}$ and $\sigma$. Lewis [12] analyzed (31) and derived the following results: the function $f(s, t, \sigma)$ is an increasing function in $\sigma$. Therefore, as $\sigma \rightarrow \infty, q^{*} \rightarrow 1$ for fixed $I^{*}=s / t$. It follows that

$$
f(s, t, \sigma)<f(s, t, \infty) \text {. }
$$

Therefore,

$$
f(s, t, \infty)=\left.\frac{-\mathscr{I}(1-\mathscr{I}) \ln (1-\mathscr{I})}{((1 / t)-\mathscr{I})[\mathscr{I}+(1-\mathscr{I}) \ln (1-\mathscr{I})]}\right|_{*}<2,
$$

is a sufficient condition for (29) to be LAS. Inequality (32) is satisfied if

$$
2\left(\frac{1}{t}-\mathscr{I}\right)[\mathscr{I}+(1-\mathscr{I}) \ln (1-\mathscr{I})]+\left.\mathscr{I}(1-\mathscr{I}) \ln (1-\mathscr{I})\right|_{*}>0 \text {. }
$$

Lewis [12] has shown that $0<s<t \leqslant 0.914$ is a sufficient condition for satisfying (33). Therefore, the polymorphic equilibrium (29) exists and is LAS if $p^{*} \sigma>1$ and $0<s<t \leqslant 0.914$ for large $\sigma$, where $s$ is not too close to $t$. The limit on $t$ extends towards 1 as $\sigma$ becomes smaller. Numerical simulations are carried out to examine the behavior of the system for large values of $t$. For $\sigma=200$ and $s=0.600$, there is a stable fixed point for $t \in(0.600,0.916]$ (e.g., for $t=0.915$ we have $(0.008,0.656)$, using the notation $\left.\left(p^{*}, \mathscr{I}^{*}\right)\right)$. For $t \in(0.916,0.946]$, there appears to be a stable 


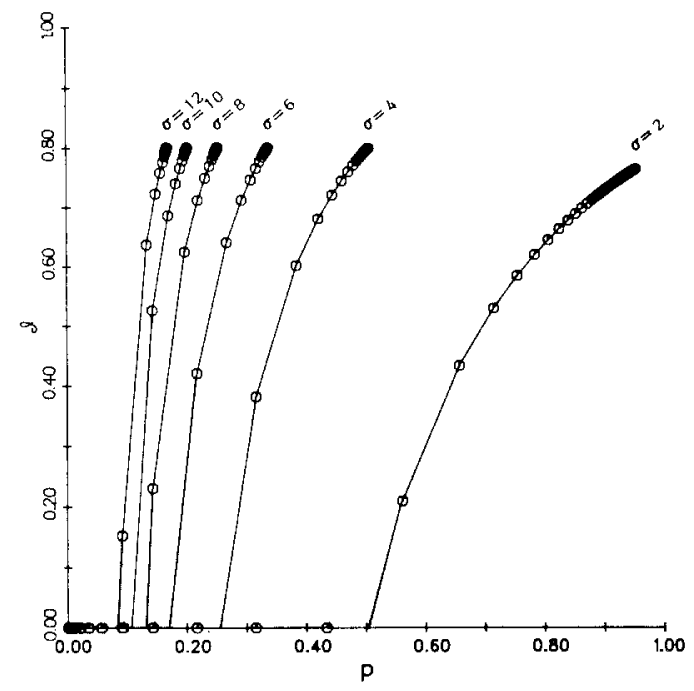

Fig. 3. Trajectories in the $p-\mathscr{I}$ plane at different values of $\sigma$, for a haploid population undergoing the $S-I-R$ epidemic process. Each trajectory starts at the initial condition $\left[p_{0}=0.01, \mathscr{H}\left(p_{0}, \sigma\right)\right]$ and moves from left to right terminating at the polymorphic equilibrium given by (26). Note that the trajectories move along the line $\mathscr{I}=0$ until the threshold $\bar{k}(p) \sigma=1$ is exceeded. Since $s / t=0.8$, the trajectories all terminate at $\mathscr{I}^{*}=0.80$. However, the same trajectories will terminate at $\mathscr{I}^{*}=s / t$ in general, provided that the conditions for (26) to be LAS are met

2-cycle (e.g., for $t=0.938$ the system cycles between $(0.010,0.799)$ and $(0.006,0.386))$. The behavior of the system is quite chaotic for $t \in(0.946,1.000)$, with no identifiable cycles. Figure 2(b) shows different stable equilibrium points for various values of $s / t$ and $\sigma$, while trajectories of the system in the $p-\mathscr{I}$ phase plane are shown in Fig. 3.

There are several points that can be made about the effect of $S-I-R$ and $S-I-S$ epidemic processes on the stability of polymorphisms. From Fig. 1, it is apparent that the $S-I-R$ process is more sensitive to changes in $p$. This sensitivity tends to be a de-stabilizing force on the system for large values of $t$, including the case where the disease is fatal, i.e., $t=1$. In contrast, the $S-I-S$ disease process is less sensitive to changes in $p$ and provides a stable polymorphism for all permissible values of $t$ and $\sigma$. The observation that $S-I-R$ epidemics tend to sweep through populations causing high attack rates, while $S-I-S$ epidemics tend to appear at constant endemic levels, lends credence to the theory that the former process is more de-stabilizing than the latter. From Fig. 2, it is apparent that the $S-I-S$ epidemic process maintains a higher frequency of the susceptible A allele than does the $S-I-R$ epidemic process, for the same population parameters.

\subsection{Diploid Population-Completely Recessive Susceptibility}

Assume that individuals of genotype $\mathbf{A A}$ are completely susceptible (i.e., $k_{11}=1$ ), then from (11),

$$
\bar{k}(p)=p^{2} .
$$


For the $S-I-S$ case, the polymorphic equilibrium is

$$
\mathscr{I}^{*}=s / t, \quad p^{*}=\sqrt{\frac{t}{(t-s) \sigma}} .
$$

The value of $p^{*}$ in the complete recessive case is the square root of $p^{*}$ in the haploid case, and is therefore greater.

Evaluating Eq. (19) yields

$$
\mathscr{I}^{\prime}=\frac{2}{p^{3} \sigma}>0, \quad \text { when } \quad p^{2} \sigma>1
$$

which upon substitution into (13) yields for the left-hand inequality

$$
\sigma^{2}(1-s)^{2}+\sigma t(2-t-s)>-t^{2},
$$

which is satisfied by condition (a) of Theorem 1 . The polymorphic equilibrium (34) is LAS if $p^{* 2} \sigma>1$ and $0<s<t \leqslant 1$, where $s$ is not too close to $t$. This result agrees with that of Kemper [11] for the continuous case. As before, Kemper has ruled at the existence of limit cycles for the continuous case.

For the $S-I-R$ case, the polymorphic equilibrium is found to be

$$
\mathscr{I}^{*}=s / t, \quad p^{*}=\sqrt{\frac{-\ln (1-I)}{\sigma I}} .
$$

The value of $\mathscr{I}^{\prime}$ is found to be

$$
\mathscr{I}^{\prime}=2 p \sigma \mathscr{I}^{2}(1-\mathscr{I})[\mathscr{I}+(1-\mathscr{I}) \ln (1-\mathscr{I})]^{-1}
$$

with $\mathscr{I}^{\prime}>0$ when $p>0$ and $\mathscr{I}<1$. Substituting (37) into (13) yields

$$
f(s, t, \sigma)=\left.\frac{-p q \mathscr{I}(1-\mathscr{I}) \ln (1-\mathscr{I})}{((1 / t)-\mathscr{I})[\mathscr{I}+(1-\mathscr{I}) \ln (1-\mathscr{I})]}\right|_{*}<1 .
$$

The function $f(s, t, \sigma)$ has a unique maximum at $p=0.5$. Therefore, the value of $\sigma$ which maximizes $f(s, t, \sigma)$ is designated $\sigma_{\max }$, and takes the value

$$
\sigma_{\max }=\frac{-\ln (1-\mathscr{I})}{0.25 \mathscr{I}}
$$

It follows that

$$
f\left(s, t, \sigma_{\text {max }}\right)=-\left.\frac{1}{4} \frac{\mathscr{I}(1-\mathscr{I}) \ln (1-\mathscr{I})}{((1 / t)-\mathscr{I})[\mathscr{I}+(1-\mathscr{I}) \ln (1-\mathscr{I})]}\right|_{*}<1
$$

is a sufficient condition for (36) to be LAS. Equation (39) is satisfied if

$$
\begin{gathered}
4\left(\frac{1}{t}-\mathscr{I}\right)[\mathscr{I}+(1-\mathscr{I}) \ln (1-\mathscr{I})]+\left.\mathscr{I}(1-\mathscr{I}) \ln (1-\mathscr{I})\right|_{*}>0 \\
4\left(\frac{1}{t}-\mathscr{I}\right) \mathscr{I}+\left(\frac{4}{t}\right)(1-\mathscr{I}) \mathscr{I}+\left.(1-\mathscr{I}) \sum_{i=2}^{\infty} \frac{4(i-1)-3 i t}{i(i-1) t} \mathscr{I}^{i}\right|_{*}>0
\end{gathered}
$$

In expression (40), the individual terms of the summation are greater than or equal 
to zero for $i \geqslant 4$. Since $0<\mathscr{I}^{*}<1$, a sufficient condition for inequality (40) to be satisfied is that

$$
\left.\frac{\mathscr{I}}{6 t}\left[24+3(4-6 t) \mathscr{I}+(8-9 t) \mathscr{I}^{2}\right]\right|_{*}>0 \text {. }
$$

The above inequality can be shown to hold for $0<s<t \leqslant 1$ using standard arguments. Therefore, the polymorphic equilibrium (36) is LAS if $p^{* 2} \sigma>1$ and $0<s<t \leqslant 1$, where $s$ is not too close to $t$. Note that $t$ has a larger bound than in the haploid case. No cycles or chaotic behavior have been found around (36) for the limits on parameters given.

\subsection{Diploid Population-Completely Dominant Susceptibility}

Assume that individuals of genotypes $\mathbf{A A}$ and $\mathbf{A a}$ are completely susceptible (i.e., $k_{11}=k_{12}=1$ ), then

$$
\vec{k}(p)=p^{2}+2 p q=p(1+q) .
$$

For the $S-I-S$ case, the polymorphic equilibrium is

$$
\mathscr{I}^{*}=s / t, \quad p^{*}=1-\sqrt{1-\theta}, \quad \text { where } \quad \theta=\frac{t}{(t-s) \sigma}<1 .
$$

In addition to condition (a) of Theorem 1, we require that $s$ not be too close to $t$ and that $\sigma$ be reasonably large in order for $0<p^{*}<1$. In this case, $p^{*}$ is smaller than the $p^{*}$ in the haploid case. Using Eqs. (15) and (19) and evaluating the left-hand inequality, we obtain

$$
(1-\theta)^{3 / 2}<\left(\frac{1-s}{t-s}\right)\left[1+(1-\theta)^{1 / 2}\right]
$$

Since $((1-s) /(t-s)) \geqslant 1$ and $0<\theta<1$, it follows that inequality (42) is satisfied for $0<s<t \leqslant 1$, using standard arguments. Therefore, (41) is LAS when $s$ is not too close to $t$. Kemper [11] has also ruled out limit cycles for the continuous counterpart of this case.

For the $S-I-R$ case, the polymorphic equilibrium is

$$
\mathscr{I}^{*}=s / t, \quad p^{*}=1-\sqrt{1-\theta}, \quad \text { where } \quad \theta=\frac{-\ln \left(1-\mathscr{I}^{*}\right)}{\sigma \mathscr{I}^{*}}<1
$$

Permissible values of $s, t$ and $\sigma$ are the same as previously defined for the completely dominant $S-I-R$ case. Using Eqs. (15) and (24) and evaluating the left-hand inequality, we obtain

$$
f(s, t, \sigma)=\left.\frac{g(p) \mathscr{I}(1-\mathscr{I}) \ln (1-\mathscr{I})}{((1 / t)-\mathscr{I})[\mathscr{I}+(1-\mathscr{I}) \ln (1-\mathscr{I})]}\right|_{*}<1,
$$

where $g(p)=p q-q /(1+q)$. The function $g(p)$ is monotone increasing in $p$ with a minimum of $-\frac{1}{2}$ at $p=0$ and a maximum of 0 at $p=1$. Therefore, the function $f(s, t, \sigma)$ has a unique maximum at $p=0$, where $g(0)=-\frac{1}{2}$, and $\sigma_{\max } \rightarrow \infty$. It 
follows that

$$
f(s, t, \sigma)<f\left(s, t, \sigma_{\max }\right)<1
$$

is a sufficient condition for (43) to be LAS. The limit of (44) as $\sigma \rightarrow \infty$ is the same as (32).

\section{Discussion}

The results of this paper are relevant to the celebrated "threshold theorem" of epidemiology [2] for the $S-I-R$ epidemic process. This theorem states that there will not be a major epidemic if $k(p) \sigma \leqslant 1$. The analogous result holds for the $S-I-S$ epidemic process where the disease will not become endemic of $\bar{k}(p) \sigma \leqslant 1$. These situations are stated by expressions (17) and (23) for the $S-I-S$ and $S-I-R$ epidemic processes, respectively. If the frequency of the susceptible $\mathbf{A}$ allele is insufficient to maintain the disease, then under certain conditions the frequency will increase through selection until the threshold is exceeded, at which time the disease will be maintained. This dynamic threshold phenomenon is shown graphically in Fig. 3. Eventually, a stable polymorphic equilibrium will be established, provided that the infection process is not too severe. Such results have been confirmed for the continuous analog of the $S$ - $I-S$ epidemic-genetic process [11]. However, in the case of a very severe infection process (i.e., the $S-I-R$ process with large $\sigma$ ) when the value of $t$ is close to one, it is possible to have limit cycles or chaotic behavior in haploid or completely dominant diploid populations. The polymorphic equilibrium is stable when $t$ is close to one in completely recessive diploid populations, even for very severe $S-I-R$ epidemics. This occurs because the density of the susceptible population is quite small, i.e., $p^{2}$; in comparison to $p^{2}+2 p q$ and $p$ for completely dominant diploid and haploid populations, respectively. A small density of susceptibles ensures that part (b) of Theorem 1 will be satisfied.

The equilibrium level of the infection $\mathscr{I}^{*}$ will depend only on the selection coefficients and not on the structure of the epidemic process, for haploid populations and diploid populations where the susceptible $\mathbf{A}$ allele is completely recessive or completely dominant. This is not true in the case of partial dominance. Assume that the fitness of an infected homozygote for the A allele is less than that of an infected heterozygote, but that they are equally fit when not infected. Then the relative fitnesses will be

$$
\begin{aligned}
& w_{11}=1-\mathscr{I} t \\
& w_{12}=1-\alpha \mathscr{I} t \\
& w_{22}=1-s,
\end{aligned}
$$

where $0 \leqslant \alpha<1$. The equilibrium value of $\mathscr{I}$, where $0<p^{*}<1$, is

$$
\mathscr{H}^{*}=\frac{s}{t}\left[\frac{q^{*}}{p^{*}+\left(q^{*}-p^{*}\right) \alpha}\right] .
$$

A good area for application is the case of $P$. falciparum and the sickle-cell trait. The correlation between the sickle-cell trait and the prevalence of $P$. falciparum has 
been well-studied [3]. In this case, we have a one locus, diallelic diploid population, where $S$ is the resistant sickle-cell allele. Then the three genotypes are $A A, A S$ and $S S$, whose individuals are totally susceptible, partially susceptible, and resistant, respectively. Then the relative fitnesses are given by (46), with $\alpha=0$ and $s=1$. Fleming et al. [7] have measured the frequency of the sickle-cell trait and the prevalence of malaria in populations of the Sudan savanna of Nigeria. They have determined the frequency of the $S$ allele to be $q=0.146$. Assuming equilibrium, they have calculated the value of $\mathscr{I}^{*} t$ as

$$
\mathscr{I}^{*} t=\frac{q^{*}}{p^{*}}=\frac{0.146}{1-0.146}=0.171
$$

One way to obtain an estimate of $\mathscr{I}^{*}$ is through the percentage of blood fields positive of $P$. falciparum. Although this varies with age and season, the zero to eight age group is most important since selection acts most strongly on the young, who have little acquired immunity. Fleming et al. [7] found the percentage of blood fields positive in this age group to average 85 percent (or $\mathscr{F}^{*}=0.850$ ). The coefficients of relative susceptibility are estimated from their data as $k_{11}=1$, $k_{12}=0.8$ and $k_{22}=0$. Evaluation of Eqs. (2) and (11) yield $\bar{k}\left(p^{*}\right)=0.929$ and

$$
\mathscr{I}^{*}=\frac{\mathscr{F} *}{\bar{k}\left(p^{*}\right)}=0.915
$$

Using $\mathscr{I}^{*}=0.915 \mathrm{in}$ (48) yields an estimate for $t$ of 0.187 . It is of interest to see if the point $\mathscr{I}=0.915, p=0.854$ is indeed an equilibrium point and, if so, whether it is LAS. To answer the latter question, we evaluate Eqs. (6) and (46), when $\alpha=0$ and $s=1$, yielding

$$
-1<1-\left.p\left[\frac{1}{p}+p t \mathscr{I}^{\prime}\right]\right|_{*}<1
$$

or

$$
\left.\mathscr{I}^{\prime}\right|_{*}<\left.\frac{3 p-1}{q p^{2} t}\right|_{*} \cong 78
$$

which must be satisfied for $\mathscr{I}^{*}=0.915, p^{*}=0.854$ to be LAS. Certainly, inequality (50) would be satisfied for most reasonable models of malaria transmission. Kemper [11] has shown that the polymorphic equilibrium for the continuous case similar to (45) coupled with the $S-I-S$ model, is LAS when $0<t \leqslant 1$. However, the epidemiology of malaria is sufficiently complex such that the simple $S-I-S$ model does not entail an adequate description of transmission. Various models have been used for malaria, although the so-called Ross-MacDonald model $[13,18]$ is still the basis for more elaborate models. Aron and May [1] give a review of the basic model. They also formulate and analyze more complex models. The derivation of $\mathscr{I}$ and $\mathscr{I}^{\prime}$ is a complex problem which will not be covered here. Clearly, an understanding of the dynamic interaction of the sickle-cell trait and P. falciparum, in indigenous populations should be an integral part of malaria control strategies.

Application of the results of this paper to specific infectious diseases requires care. As previously mentioned, the $S-I-S$ and $S-I-R$ epidemic processes are only 
rough approximations to actual disease processes. A more realistic host population with age structured susceptibility and overlapping generations would undoubtedly be necessary for application of the model. Inclusion of overlapping generations would necessitate the temporal rather than the asymptotic analysis of the epidemic equations, since the exposure of individuals to infection would no longer be uniform across generations. In addition, such considerations as genetic drift and migration would have to be considered in some cases, e.g., migration plays an important role in maintaining foci of infection during the consolidation phase of malaria eradication programs [19]. Nevertheless, the simple models presented here should provide insight into the dynamic interaction of the host genotype and infectious disease.

Acknowledgements. I am most grateful to Dr. John Kemper, who has been helpful in clarifying several mathematical points in this paper. I am also grateful to Drs. Peter Smouse and Alexander Gimelfarb for their constructive criticism.

\section{References}

1. Aron, J. L., May, R. M.: The population dynamics of malaria. Population dynamics of infectious diseases. Anderson R. (ed.), pp. 139-179. Chapman and Hall, Ltd. 1981

2. Bailey, N. T. J.: The mathematical theory of infectious disease and its application (2nd ed.). New York: Hafner Press 1975

3. Birdsell, J. B.: Human evolution. Chicago: Rand McNally 1972

4. Bremermann, H. J.: Sex and polymorphism as strategies in host-pathogen interactions. J. Theoret. Biology 87, 671-702 (1980)

5. Cockerham, C. C., Burrows, P. M., Young, S. S., Prout, T.: Frequency dependent selection in randomly mating populations. Amer. Nat. 106, 493-515 (1972)

6. Crow, J. F., Kimura, M.: An introduction to population genetics theory. New York: Harper and Row 1970

7. Fleming, A. F., Storey, J., Molineaux, L., Iroko, E. A., Attai, E. D. E.: Abnormal haemoglobins in the Sudan savanna of Nigeria. Ann. Trop. Med. Parasit. 73, 161-173 (1979)

8. Gillespie, J. H.: Natural selection for resistance to epidemics. Ecology 56, 493-495 (1975)

9. Hethcote, H. W. : Qualitative analyses of communicable disease models. Math. Biosci. 28, 335-356 (1976)

10. Jayakar, S. D.: A mathematical model for interaction of gene frequencies in a parasite and its host. Theoret. Population Biology 1, 140-164 (1970)

11. Kemper, J. T.: The evolutionary effect of endemic infectious disease: Continuous models for an invariant pathogen. J. Math. Biol. 15, 65-77 (1982)

12. Lewis, J. W. : On the coevolution of pathogen and host: II. Selfing hosts and haploid pathogens. J. Theoret. Biology 93, 953-985 (1981)

13. MacDonald, G.: The analysis of equilibrium in malaria. Trop. Dis. Bull. 49, 813-828 (1952)

14. Mathews, H. M., Armstrong, J. C.: Duffy blood types and vivax malaria in Ethiopia. Am. J. Trop. Med. Hyg. 30, 299-303 (1981)

15. Miller, L. H., Steven, J. M., Clyde, D. F., McGinniss, M. H.: The resistance factor to Plasmodium Vivax in blacks. New Eng. J. Med. 295 (1976)

16. Molineaux, L., Gramiccia, G.: The Garki project. Geneva: World Health Organization 1980

17. Rosenstreich, D. L.: Genetics of resistance to infection. Nature 28, 436-437 (1980)

18. Ross, R.: The prevention of malaria (2nd ed.). London: Murray 1911

19. Yekutiel, P.: Problems of epidemiology in malaria eradication. Bull. Wld. Hlth. Org. 22, $669-683$ (1960)

20. Yu, P.: Some host parasite genetic interaction models. Theoret. Population Biology 3, 347-357 (1972)

Received June 1982 